\title{
DIE STUDENTENBEWEGUNG 1968 IM SPIEGEL DER DEUTSCHEN PRESSE. EIN ÜBERBLICK ÜBER DIE SCHLAGZEILEN ZUR ANTI-SPRINGER-KAMPAGNE IM APRIL 1968
}

\author{
JACEK SZCZERBAK \\ Adam Mickiewicz University - Poznań
}

\begin{abstract}
This article describes the attitudes of German press to the events of the student protest in West Berlin and West Germany in 1968. A number of headlines from five major German newspapers and magazines (from Das Bild to Neues Deutschland) have been chosen to illustrate how the same facts were differently interpreted by left-wing and right-wing journalists. The language of political propaganda is in the author"s focus of attention although the first part of the article also covers some historical aspects of the student revolt. Since the media played an active role in the revolt on both sides of the social conflict, this short analysis of the German press might be of interest to linguists and historians alike. Among the analyzed linguistic aspects of the headlines are the following: the degree of neutrality or objectivity vs. emotional bias, the use of quotations, irony, colloquialisms as well as the perspective and the content of the headlines as speech acts. On this basis the author tries to define the position of the five newspapers and magazines in the political debate about the events of 1968 which continues to this day.
\end{abstract}

Der Zusammenhang zwischen Politik und Sprache fasziniert wohl seit der Erscheinung von 1984 von George Orwell sowohl Schriftsteller und Publizisten als auch Wissenschaftler, darunter Historiker, Medienforscher und Linguisten. Die politische und wirtschaftliche Spaltung in den kapitalistischen Westen und den kommunistischen Osten nach dem 2. Weltkrieg vollzog sich auch auf dem Gebiet der Sprache, besonders der politisch relevanten Mediensprache, was nirgendwo deutlicher zu spüren war als im geteilten Deutschland. Der unterschiedliche Sprachgebrauch, auf den Reich anhand von Beispielen aus dem Leipziger und dem Mannheimer Duden hinweist (Reich 1968:269), sowie die unterschiedliche Auffassung politischer Wirklichkeit in ost- und westdeutschen Medien, machte viele Forscher auf das Phänomen der Propagandasprache aufmerksam. Im Zentrum des Interesses stand die Sprache des III Reiches, die als ein mächtiges politisches Instrument aufgefasst wurde, das nicht zuletzt zum Erfolg der NaziIdeologie beitrug und der NSDAP zur Macht verhalf. Ein nennenswerter Titel ist in diesem Zusammenhang LTI (Lingua Tertii Imperii) - Notizbuch eines Philolo- 
gen von Viktor Klemperer, der auf der Grundlage persönlicher Beobachtungen die sprachlichen und psychologischen Mechanismen der Nazi-Sprache aufdeckt.

Mit dem totalitären Sprachgebrauch des Naziregimes wurde in Westdeutschland oft die stark ideologisierte Sprache der DDR verglichen (vgl. Gudorf 1981:19-21), in Ostdeutschland dagegen haben Sprachforscher Manipulationen in den westlichen Medien, unter anderem in der Werbung, kritisiert (vgl. Bahner: Imperialistische Manipulation und Sprache). Die politischen oder ideologischen Positionen, aus denen geforscht und argumentiert wurde, beeinträchtigen die wissenschaftliche Qualität vieler dieser Beiträge, sie zeugen aber zugleich von der engen Beziehung zwischen Sprache und Politik, auch in der Wissenschaft.

Besonders deutlich trat diese Beziehung zur Zeit der APO (Außerparlamentarische Opposition) um das Jahr 1968 in den Vordergrund. Die sich über Westberlin und westdeutsche Universitätsstädte erstreckende Studentenbewegung wurde nicht nur unterschiedlich in der BRD und der DDR rezipiert, sondern führte auch zu einer starken politischen Polarisierung innerhalb westdeutscher Medien, vor allen Dingen in der Presse. Sie geriet in den Sog politischer Auseinandersetzungen, die auch die deutsche Gesellschaft z.T. spalteten. Dabei wurde die Rolle der Massenmedien von der Protestbewegung selbst thematisiert und eine intensive Medienarbeit betrieben. Deshalb bietet die Presse aus dieser Zeit reichlich Material zu Untersuchungen des Verhältnisses zwischen Sprache und Politik.

Die Träger der Protestbewegung 1968 verstanden sich oft als die „revolutionäre Klasse", die nach dem Vorbild der Befreiungskriege in der Dritten Welt die westdeutsche Gesellschaft von „repressiven kapitalistischen Machtverhältnissen” befreien soll. Ihr Protest richtete sich nicht nur gegen autoritäre Universitätsstrukturen und Zulassungsbeschränkungen (,Numerus Clausus“), gegen den von der Großen Koalition gebilligten Vietnamkrieg, gegen die zu verabschiedenden Notstandsgesetze, sondern gegen den Lebensstil und die Moralvorstellungen der deutschen Nachkriegsgesellschaft schlechthin. Man forderte mehr als Reformen; viel mehr war man von der Idee einer „Kulturrevolution” nach dem chinesischen Muster begeistert. Zu den Leitfiguren der Protestbewegung zählten Martin Luther King, genauso wie Che Guevara und Mao Tse Tung.

Eine eindeutige linkspolitische Tendenz in der Bewegung ging mit der Abgrenzung von der älteren Generation einher, der die Duldung des Nazi-Regimes vorgeworfen wurde. Angesichts des Vietnam-Krieges, der mit den Massenmorden der Nazi-Zeit verglichen wurde, wollte die 68-er Generation den Fehler ihrer Väter nicht wiederholen und untätig bleiben (vgl. Krebs 1988:149); eine ihrer Demonstrationsparolen war: „Bürger, lasst das Gaffen sein!“ (vgl. Halter). Mit Parolen dieser Art, wie: „Unter den Talaren Muff von Tausend Jahren“ (über konservative, prestigebewusste Professoren), „Notstandsterror“, „Lieber rot als tot" (in bezug auf Vietnam) und „Vertrau keinem über dreißig" versuchten sie, die Öffentlichkeit anzusprechen.

Dabei wurden neben Politikern, Professoren und Eltern auch Journalisten von ihrer Kritik nicht verschont. In erster Linie wurde der Springer-Konzern angegriffen, der mit seinem Marktanteil von 80\% in Berlin (Mager 1968:44) einen 
erheblichen Einfluss auf die öffentliche Meinung ausübte. Die starke rechte Tendenz der Springerschen Boulevardblätter (Bild, Berliner Morgenpost) wie auch der Qualitätszeitung Die Welt wurde von den linksorientierten Studenten als eine Gefahr der ,Renazifizierung" gesehen. Auf manchen Transparenten (Losung: "Springer - Mörder") bei der Anti-Springer-Kampagne wurde der Name Springer mit 2 blitzförmigen „S“ in Anlehnung an das Nazisymbol SS dargestellt (ND 13/14.04.1968:4).

Die Rolle der Massenmedien für die Schaffung und Steuerung der Öffentlichkeit wurde von der APO frühzeitig erkannt, was sich in ihren Forderungen nach Sendezeit in öffentlichen Medien widerspiegelte (ND 13/14.04.1968:3), sowie in der spektakulären Form vieler Aktionen, die das Interesse der Medien erwecken und damit ein breites Publikum erreichen sollten. Dabei wurden die Medien von den Studenten oft funktionalisiert, indem sie manche Aktionen als Medienereignisse inszenierten. Rainer Langhans, Mitglied der Kommune 1, die ihren persönlichen Protest mittels alternativer Lebensweise und politischer Happenings zum Ausdruck brachten, berichtet:

Wir wussten auch bald, wie man mit dem Fernsehmedium umzugehen hat. Wir wollten nicht spießig sein und vor allem unberechenbar bleiben. Das war unsere subversive Medienarbeit. (zit. nach Jansa 1999:191)

Als unberechenbar kann man mit Sicherheit das berühmte „Puddingattentat” auf den US-Vizepräsidenten Humphrey bezeichnen, oder die Fahrt des Kommunarden Fritz Teufels auf einem Kinderfahrrad im Ornat und mit der Amtskette des Rektors der FU (vgl. Jansa 1999:173). Ziel ähnlicher Aktionen war, Autoritäten lächerlich zu machen, und durch Spaß an gemeinsamer Handlung die Protestierenden zu solidarisieren. Zugleich dienten sie der Werbung um Nachfolger und Sympathisanten, die eine „Gegenöffentlichkeit“ bilden sollten.

Nicht immer konnte sich die Führung der Protestbewegung auf die Medien verlassen. Die konservativen und regierungsnahen Springerblätter waren der Bewegung kritisch, ja feindselig gestimmt. Nach dem „Puddingattentat“ erschienen u.a. folgende Schlagzeilen: „Geplant - Berlin: Bombenanschlag auf US-Vizepräsident" (Bild), „Maos Botschaft in Ost-Berlin liefert die Bomben gegen VizePräsident Humphrey“ (Der Abend), „Attentat auf Humphrey von Kripo vereitelt FU-Studenten fertigen Bomben aus Peking" (Berliner Morgenpost) (zit. nach Krebs 1988:130). Die Pläne, Humphrey mit Pudding, Mehl und Farbe zu bewerfen, wurden zu einer mörderischen Verschwörung stilisiert. Ob es aus Unwissen oder absichtlich geschah - die Studenten sahen ihre These von der anti-studentischen „Hetze“ des Springer-Konzerns bestätigt (vgl. 1968:46). Selbst in seriösen und nicht Springerschen Blättern wurden zuweilen radikale Ansichten vertreten, die den radikalen Charakter der politischen Auseinadersetzungen von 1968 widerspiegeln. Der Frankfurter Rundschau veröffentlichte zum selben Ereignis folgenden Kommentar: 
Wer glaubt, sich nur mit Explosionskörpern [lies: Pudding - J.S.] Gehör verschaffen zu können, muss sich gefallen lassen, dass er zu jenen gerechnet wird, die sich der Sprache der Bomben verpflichtet fühlen. (zit. nach Krebs 1988:132)

Nicht selten waren diese Aktionen zielbewusste Provokationen, vor allem an die Polizei gerichtet, mit dem Ziel, die Brutalität der Staatsgewalt zu ,entlarven“ und durch Mitleid Sympathie der Öffentlichkeit zu gewinnen. Nach einem Flugblatt von SDS (Sozialistischer Deutscher Studentenbund) soll man auf diese Weise „der latente Faschismus in offenen Faschismus umschlagen lassen“ (zit. nach Brandt 1986:68), und in der Gesellschaft eine Empörung über den „faschistischen" Staat zu erwecken. Ein Beispiel für eine phantasievolle Öffentlichkeitsarbeit kann die „Spaziergängerdemonstration“ liefen, die am verkaufsoffenen Adventssonnabend 1967 veranstaltet wurde. Die Demonstranten hatten sich als Weihnachtseinkäufer mit Geschenkpaketen getarnt. Immer wenn die Polizei erschien, um die Demonstration zu zerschlagen, ertönte eine Kindertrompete und die Demonstranten mischten sich unter die Passanten. In Folge dieser Taktik wurden die Polizisten so desorientiert, dass sie auch zufällige Passanten schlugen (vgl. Krebs 1988:132). Wer die Staatsgewalt am eigenen Leib erfuhr, war auch eher dazu geneigt, die von der APO geübte Kritik des Staatsapparates für richtig zu halten.

Jansa nennt die wachsende Rolle der Massenmedien in den 60er Jahren einen wichtigen Faktor für die Entstehung und Form der Protestbewegung (1999:190-199). Ohne die Fernsehberichte vom Vietnamkrieg, die Millionen Zuschauer seine Grausamkeit vor Augen führte, wäre eine derart starke Betroffenheit und Mobilisierung der Öffentlichkeit dagegen nicht möglich. Mancher Fernsehzuschauer oder Zeitungsleser muss auf den starken Kontrast zwischen diesen Bildern und vielen tendenziösen proamerikanischen Kommentaren aufmerksam geworden sein. Es wurde sogar behauptet, in Vietnam verteidige Amerika die Freiheit Berlins. Die offizielle Linie der Berichterstattung bringen die Worte eines deutschen Vietnam-Korrespondenten zum Ausdruck:

...schließlich können wir Deutsche, die wir den Amerikanern so viel verdanken, diesen Amerikanern nicht gut in den Rücken fallen. Bei jeder Zeile, die ich schreibe, muss ich an die große Verantwortung denken, die ein Journalist trägt. Deshalb konnte ich das, was ich Ihnen hier mündlich erzählt habe, nicht schreiben. (zit. nach Glotz 1969:40)

Diese aus politischer Rücksicht oder Sympathie vorgenommene Selbstzensur von Teilen deutscher Journalisten, bewirkte vor allem bei der jungen Generation das Gegenteil vom Beabsichtigten: statt ein Verständnis für die amerikanische AuBenpolitik zu erzeugen, bestätigte sie ihre Überzeugung, dass in Nachkriegsdeutschland genauso wie in der Nazi-Zeit Völkermord offiziell geduldet, ja unterstützt wird. Viel stärker als politisch rücksichtsvolle Kommentare wirkten wohl 
die Fernsehbilder von Zerstörung des Landes und Leiden der Zivilbevölkerung oder bloße Fakten wie Zahlen der Toten, die die Kommentare in Frage stellten. Herbert Marcuse, der zu den wichtigsten Theoretikern der Protestbewegung gezählt wird, hat die Berichte über Vietnam in amerikanischer Presse, u.a. Ausdrücke wie „killing rate“, als Zeichen von „Dehumanisierung“ westlicher Gesellschaft gedeutet.

So wie der Kalte Krieg, der in den 60er Jahren sehr intensiv geführt wurde, ein psychologischer Propagandakrieg war, fand die Studentenrevolte nicht nur auf der Straße statt, sondern in erster Linie in den Medien und im Bewusstsein der Menschen. Berndt Rabehl, der zusammen mit Rudi Dutschke zu der SDSFührung gehörte, erinnert sich:

Wir haben gesagt, das ist keine revolutionäre Situation, sondern eine vorrevolutionäre. Wir gehen nicht in die Illegalität, um nicht den gesamten Gewaltapparat auf uns abzulenken, da haben wir keine Chance. Wir sind sozusagen in unserer Phantasie, in unserer Provokation stärker als die Polizei, aber nicht in den technischen Auseinandersetzungen mit der Polizei. Wir müssen die Formen nehmen, wo wir stärker sind: das ist in der Propaganda, in der Provokation, in der Agitation, in der Demonstration, in der Mobilisierung, in der Phantasie. (zit. nach Krebs 1988:147)

In der deutschen Presse wurde ein Meinungskampf zwischen verschiedenen Interessengruppen geführt, der eine starke Politisierung der Berichterstattung und der Zeitungssprache selbst zur Folge hatte. Sensationsgierige Boulevardblätter bekämpften die Protestbewegung mit einer rücksichtslosen Propaganda-Sprache, die linke Presse griff zu Kommunismus-Klassikern und der Terminologie des „Klassenkampfs", andere Redaktionen bemühten sich um ein unparteiisches, ideologiefreies und sachliches Journalistendeutsch. Dazu haben unterschiedliche Zeitungen aus unterschiedlichen Perspektiven, mit unterschiedlichen Schwerpunkten und sogar von unterschiedlichen Tatsachen berichtet, die sie im Zusammenhang mit der Protestbewegung erwähnten und kommentierten; andere Tatsachen wurden auch verschwiegen. Da all die Zeitungen und Zeitschriften mit ihrer Version der Ereignisse Tausende oder Millionen Leser beeinflussten, indem sie diese oder jene Tatsachen und Meinungen verbreiteten, und da die Protestierenden selbst mit Aktionen, Flugblättern oder Publikationen die Öffentlichkeit für ihre Revolte gewinnen wollten, kann die Rolle der Medien für die Protestbewegung kaum überschätzt werden.

Neben historischen Beiträgen kann auch eine sprachorientierte Textanalyse der Zeitungsartikel, die den studentischen Protest begleiteten, wesentlich zum Verständnis der Ereignisse von 1968 und der Haltungen der deutschen Gesellschaft beitragen. Im Rahmen dieses Beitrags soll nur ein kleiner Zeitraum von den Tagen nach dem Attentat auf Rudi Dutschke, den unoffiziellen Führer des SDS, untersucht werden. Diese Wahl wurde aus mehreren Gründen getroffen: zum einen hat dieses Ereignis zu einer Welle von Protesten geführt, die als der 
Höhepunkt der Protestbewegung, oder zumindest seiner antiautoritären Phase, gilt (vgl. Jansa 1999:69, 74). Zum anderen wurde im Kontext des Mordanschlags auf Dutschke über die Rolle der Medien im politischen Leben intensiver als je diskutiert, was sich u.a. in der Anti-Springer-Kampagne äußerte. Ferner hatte das Attentat eine noch deutlichere Verschärfung der politischen Tendenzen in der Berichterstattung zu Folge, so dass sie leicht erkennbar sind.

Zur Analyse wurden Texte aus drei Tageszeitungen und zwei Zeitschriften unterschiedlicher Qualität und politischer Tendenz gewählt. Diese Auswahl sollte dem Leser ein möglichst breites Spektrum von zuweilen extremen journalistischen Stilen und Haltungen präsentieren: die Bild-Zeitung, das von der Studentenbewegung am meisten gehasste Ziel der Anti-Springer-Kampagne, die sogar zusammen mit anderen Springerblättern für das Attentat auf Rudi Dutschke, wie auch für die Erschießung des Studenten Benno Ohnesorg am 2. Juni 1967 verantwortlich gemacht wurde; die Süddeutsche Zeitung als Beispiel von liberalen Haltungen und professionellem Journalismus; Neues Deutschland, das wichtigste Organ der SED, das Aufschluss über die offizielle ostdeutsche Version der Ereignisse geben soll; das Magazin konkret, das zu dieser Zeit als ein Forum linker Aktivisten und Intellektueller galt; schließlich das sowohl dem Staat als auch den Studenten gegenüber ironisch-kritische, unabhängige Magazin Der Spiegel. Zur Rolle und Spezifik von Überschriften hat sich Happenkamps folgendermaßen geäußert:

An Schlagzeilen können Merkmale einer „Selektionssemantik“ besonders gut aufgezeigt werden. Durch die Wahl der Schlagzeilen wird zum einen ein bestimmter inhaltlicher Aspekt hervorgehoben, zum anderen werden durch die besondere Kennzeichnung dieses Aspekts (Konnotationen) eigene Einstellungen angezeigt. (Happenkamps 1979:187)

Zwar bilden die keine ausreichende Basis für eine systematische Textanalyse, doch können sie ein interessanter Ausgangspunkt für Überlegungen zur Pressesprache bilden. Es sind - neben Fotos - zunächst die Überschriften, die die Aufmerksamkeit des Lesers auf einen Artikel lenken. Aus dieser Werberolle ergibt sich eine besondere Prägnanz der Formulierung, die oft die Perspektive oder Einstellung des Autors erkennen lässt. Außerdem entscheidet eine Schlagzeile erst darüber, ob ein Artikel überhaupt gelesen wird oder nicht; nach neuesten Untersuchungen gibt die Hälfte der Zeitungsleser schon nach der Überschrift zu lesen auf, ein Viertel nach dem ersten Satz (vgl. Linden 2000:8). Wenn man also von der meinungsbildenden Rolle der Presse spricht, dann beschränkt sich diese für viele auf die Wirkung von Schlagzeilen. Darüber hinaus können im Rahmen dieses Beitrags - ihrer Kürze wegen - sämtliche Überschriften vom Zeitraum nach dem Mordanschlag auf Rudi Dutschke zitiert werden, was einen Überblick über die Themenauswahl und den Stil der Berichterstattung ermöglichen soll. Aus diesen Gründen werden hier die Schlagzeilen als ein wichtiges Textelement untersucht. 
Süddeutsche Zeitung veröffentlichte vom 13. bis zum 20. April 1968 zum Attentat auf Rudi Dutschke und den danach folgenden Protesten Artikel unter folgenden Überschriften:

(1) Nach dem Mordanschlag auf Rudi Dutschke eine Welle von Protestdemonstrationen

(2) Kiesinger mahnt zu Besonnenheit

(3) Der Attentäter ,ein guter, braver Junge“

(4) Nach dem Attentat Steine gegen das Glashaus

(5) Demonstranten stürmen „Bild“-Redaktion

(6) Sympathie für den Studentenprotest

(7) Die Ausschreitungen alarmieren Bonn. Sondersitzung des Innenausschusses

(8) Ostermarschierer rufen zu Aktionen auf

(9) Politiker um einen Brückenschlag bemüht

(10) Schlägerei vor der deutschen Botschaft in London

(11) Nach der Gewalt die Besinnung

(12) Strafanzeige der Frankfurter Societäts-Druckerei

(13) Sondersitzung des Bundestags. Tagesordnung: die Unruhen

(14) Aus der TU Rufe nach Gewalt

(15) Statt Spaziergänge die „direkte Aktion“

(16) Mit roter Fahne und Dutschke-Bildern...

(17) Bundesregierung will Grundrechte schützen und Gewaltanwendung unterbinden

(18) Pressephotograph Opfer der Krawalle

(19) Albertz um Vermittlung bemüht

(20) Mit dem Herzen auf Seiten der Studenten

(21) Wer warf den tödlichen Stein?

(22) Künftig notfalls auch mit Schlagwaffen

(23) Berliner Studenten sind uneins

(24) Zweites Opfer der Münchner Krawalle

(25) Bürgermeister appelliert an die Berliner

(26) Mit Gewalt die Welt verbessem?

(27) SDS-Sprecher: Nicht rührselig werden

Eine wichtige Eigenschaft dieser Überschriften - und ein Vergleich mit den anderen Zeitungen und Zeitschriften wird diese Erkenntnis bestätigen - ist ihre nüchterne Neutralität und ein relativ großer Informationswert. Bis auf (7), (14), (16) und (18), (24) enthalten sie keine Wertung; statt an Emotionen zu appellieren, sollen sie möglichst sachlich den Leser über die Ereignisse informieren. Bei (7), (14), (16) und (18), (24) ist jedoch eine negative Wertung der Proteste sichtbar. Die Wörter „Ausschreitungen“ (7), in größerem Maße „Krawalle“, auch „Opfer“ (18), (24) haben deutlich negative Konnotationen. (Hier wird "Schlägerei“ (10) nicht erwähnt, weil diese Bezeichnung sich nicht direkt auf die deutsche Studentenbewegung bezieht und sowohl deren englischen Sympathisanten als auch der englischen Polizei zugeschrieben werden kann.) Bei (16) signalisieren die drei Punkte eine ironische Einstellung des Autors, auch im Audimax der TU wurde 
wahrscheinlich nicht nach „Gewalt" (14), sondern eher „Aktion" gerufen, wie bei (15). Da wurde die Sprache der Protestierenden übernommen, obwohl die Anfuihrungszeichen eine Distanz, bzw. Ironie markieren. Eine Aussage der Regierung wird dagegen ohne diese Distanz zitiert - (17). Eine Regierungsnähe ist auch daran deutlich, dass die Handlungen von Politikern, die zur Schlichtung des Konflikts führen sollten, mehrmals thematisiert werden - (2), (9), (19), nicht aber ihre Unterstützung der strengen polizeilichen Mittel gegen Demonstranten, wie bei konkret oder Der Spiegel. Die Demonstranten werfen Steine (4), (21) und rufen zu Aktionen/Gewalt - (14), (15) auf; von ihren Zielen jedoch erfährt der Leser zumindest von den Überschriften - weniger als von den Zielen der Regierung. Diese Tendenz der Presse, von den äußeren Erscheinungen des Protests viel ausführlicher als von seinen Inhalten zu berichten, wurde oft seitens der Studentenbewegung formuliert (vgl. Mager 1968:46). Die negative Haltung der Süddeutsche Zeitung der Protestbewegung gegenüber ist auch daran sichtbar, dass sie fast immer - (4), (5), (11), (14), (17), (24), (26) - bis auf (23) und (27) - nur mit Gewalt in Zusammenhang gebracht wird, dabei wird außer Acht gelassen, was im Spiegel und in konkret mehrmals betont wird - dass die Gewalt oft von der Polizei kam. In (22) wird das Gegenteil behauptet.

Trotzdem bleibt Süddeutsche Zeitung relativ objektiv, indem sie von der Solidarisierung von englischen Ostermarschierern - (10) und des Fotografen Klaus Frings, der während einer Münchner Demonstration durch Steinwurf getötet wurde - (20) mit den Studenten berichtet. Neben Politikern - (2), (19), (25) und einem Polizei-Sprecher - (22) wird auch der SDS-Sprecher zitiert - (27); auf diese Weise bietet die Zeitung ein Forum für alle beteiligten Parteien und ermöglicht ihrem Leser, über die Ereignisse ausführlich und aus unterschiedlichen Perspektiven zu erfahren.

Als Kontrast zu Süddeutsche Zeitung mögen folgende Überschriften aus Neues Deutschland (vom 13. bis zum 16. April 1968) dienen:

(1) Mordanschlag auf sozialistischen Studentenführer

(2) Die Saat der Renazifizierung geht auf (ganze Seite, invers)

(3) Ein Anschlag auf alle Notstandsgegner

(4) Die Blutspur vom Freitag, 2. Juni, zum Donnerstag, 11. April

(5) Anklage gegen System der Renazifizierung

(6) So hetzten die Hintermänner zum Mord (invers)

(7) So heucheln die Hintermänner nach der Tat (invers)

(8) Karfreitag, 15.30 Uhr, in Westberlin...

(9) Berichte von den Demonstrationen gegen den Notstandsterror (ganze Seite, invers)

(10) Empörung und Protest gegen Mordterror

(11) Leitung des Terrors: Kiesinger persönlich

(12) Lebende Mauer gegen Springers Autos

(13) Entrüstung im Ausland

(14) Antifaschistische Einheitsfront in Westberlin gebildet 
(15) Blut-Ostern in Westberlin

(16) Massenproteste in Westdeutschen Stådten

(17) Notstand und Aggression am Pranger

(18) Strauß und Benda gossen Mordkugeln

(19) Imperialismus zeigt sein wahres Gesicht

(20) Empörung in ganz Europa

(21) Arbeiterfunktionäre: Alarmzeit für uns alle

(22) Dokumente vom Kampf der demokratischen Kräfte (ganze Seite, invers)

(23) Bonn erprobt in Westberlin Notstandsdiktatur

(24) Ostern 1968 - Kampagne gegen Renazifizierung

(25) Benda: Verschärfung des Terrors

(26) Bisher größte Protestaktionen der Notstandsgegner

(27) Schütz dankt Knüppelgarde

(28) Offenbarung eines mörderischen Systems

(29) Grausamkeiten einer entfesselten Soldateska

(30) Machtvoller Abschluss der Ostermärsche

(31) Berichte vom Kampf gegen Renazifizierung und antidemokrat. Terror (ganze Seite)

(32) Solidarität mit den Kräften der Demokratie (ganze Seite, invers)

Neues Deutschland berichtet ausführlicher als Süddeutsche Zeitung von der Protestbewegung, wobei außer Überschriften, die sich auf einen bestimmten Text beziehen, werden auch invers (weiß auf schwarz) geschriebene Schlagzeilen gedruckt, die für die ganze Seite mit mehreren Artikeln gelten. Am Vergleich zwischen diesen zwei Zeitungen sieht man einen krassen Unterschied zwischen der west- und ostdeutschen Perspektive, oder zumindest der SED-Perspektive, die als offiziell galt. Statt Zurückhaltung - Anteilnahme, statt Sachlichkeit - Leidenschaft. Die Bezeichnung „Protestaktionen“, die von der Studentenbewegung selbst stammt, wird entweder von Neues Deutschland übernommen: „Protest" (10), „Kampf“ - (24), „Protestaktionen“ - (26) oder durch den euphemistischen Ausdruck: „Empörung“ ersetzt - (10), (20). Dabei werden diese Aktionen ziemlich heroisch dargestellt - (12); nicht nur wird auf ihren massenhaften Charakter hingewiesen - (16), (20), (30), sondern wird auch ihr Ziel, d.h. der abstrakte Feind so formuliert, dass jeder Neues-Deutschland-Leser sich mit den Protesten identifizieren soll. Wer ist schon nicht gegen "Terror" - (11), (25), geschweige denn einen „Notstandsterror“ - (9) oder gar „Mordterror“ - (10)? In diesen Überschriften wird der politische Konflikt zwischen der APO und der Bonner Regierung zu einem militärischen Konflikt zwischen Demokraten und Nazis stilisiert. Ausdrücke aus dem Militärbereich kommen in folgenden Überschriften vor - (3), (12), (14), (22), (27), (29), (31).

Außer den oben genannten Bezeichnungen für die Proteste wird auch von der Sprache der Protestbewegung der politische Schüsselbegriff „Renazifizierung" entlehnt - (2), (5), (24), (31). Die andere Seite darf zwar auch zu Wort kommen, doch ihre Aussagen werden schon in der Überschrift als "Heuchelei“" disqualifiziert - (9), oder ihre Autoren werden als „Hintermänner“ diffamiert. 
Hier wird den zitierten Bonner Politikern eine böswillige Absicht unterstellt und eine der journalistischen Hauptregeln - die Regel der Objektivität der Zitate verletzt (vgl. Happenkamps 1979:58). An Stellen, wo sie zitiert werden, tauchen Formulierungen auf, die die Zitierten mit Sicherheit nicht verwendet hätten; Benda kündigte wahrscheinlich eine „Verschärfung der polizeilichen Maßnahmen“, nicht „Verschärfung des Terrors" - (25) an. Dasselbe trifft für (27) zu; in seiner Danksagung an die Polizei kann Schütz keine Invektiven verwendet haben.

In den politischen Invektiven, die der Großen Koalition gelten, ist Neues Deutschland den Studenten selbst voraus; wo sie über das "repressive System“ klagten, schreibt Neues Deutschland "mörderisches System" - (28). Auch Bezeichnungen wie „entfesselte Soldateska“ - (29) sind selbst in den radikalsten Aussagen der Protestierenden nicht zu finden. Obwohl die SED-Zeitung eindeutig auf die Protestierenden Partei ergreift, ist ihre Perspektive anders als die der APO. Es hängt zwangsläufig damit zusammen, dass die Unruhen 1968 nur Westdeutschland und Westberlin betrafen; von der DDR aus wurden sie also als Auslandsereignisse betrachtet. Trotz dieser Parteinahme für die Studentenbewegung wird sie - was schon den Überschriften zu entnehmen ist - von Neues Deutschland weitgehend funktionalisiert und für eigene, ostdeutsche, politische Ziele benutzt. Die Anti-Springer-Kampagne der Studentenbewegung wird zu einer AntiBonner-Kampagne von Neues Deutschland umfunktioniert, was den oben zitierten politischen Invektiven zu entnehmen ist. Selbst Süddeutsche Zeitung, die den Protesten gegenüber kritisch gestimmt ist, berichtet mehr von deren Zielen und Hintergründen - (8), (26), als Neues Deutschland, wo die diffusen Ziele der Bewegung noch diffuser und unschärfer definiert werden - (5), (10).

Auffällig ist der starke affektive Charakter der Überschriften; im Vergleich mit Süddeutsche Zeitung haben sie einen geringeren informativen Wert, denn ihre Funktion ist nicht zu informieren, sondern zu mobilisieren, zu entsetzen. Besonders gut eignen sich dafür quasi literarische Ausdrücke wie die zwei Zusammensetzungen mit „Blut“: „Blutspur“ - (4) und „Blut-Ostern“- (15). Auch Metapher - (2), (18) und eine Personifizierung des Imperialismus - (19) werden dem Leser nicht erspart. Anders als in Süddeutsche Zeitung enthält die Mehrheit der Überschriften von Neues Deutschland eine klare Wertung der Ereignisse oder der Beteiligten; nur (1), (8), (13), (16), (17) und (20) können als neutral gelten.

Die auch linke, aber westdeutsche Monatszeitschrift konkret veröffentlichte in der Aprilnummer 1968 folgende Artikel:

(1) Vom Protest zum Widerstand

(2) Staat der Gewalt. Bluthunde, Tränengas, Knüppel

(3) Protokoll eines Attentats

(4) Treibjagd auf Demonstranten

(5) Eine neue Phase des Kampfes

(6) ,Enteignet Springer“. Warum?

(7) Gewalt 
Eine Unparteilichkeit ist im Fall von konkret, wegen der Nähe der Redaktion zur APO, kaum zu erwarten, z.B. Ulrike Meinhof, Autorin von (1), kannte Rudi Dutschke persönlich und schrieb aus persönlicher Betroffenheit. Von der Teilnahme der Zeitschrift am Protest berichtet Krebs:

konkret gibt nicht nur die Stellungnahmen der studentischen Ausschüsse heraus und berichtet über deren Aktionen, sondern greift mit Interviews, Artikeln und Sonderdrucken in die studentischen Aktivitäten ein. (Krebs 1988:56)

Trotzdem bemüht sich konkret um neutrale Überschriften; nicht neutral sind hier die zwei, die die Brutalität der Polizei zum Thema haben - (2) und (4). In (2) wird dem Leser diese Brutalität durch das Nennen von polizeilichen Gewaltmitteln als eine sinnlich erfassbare Erfahrung vermittelt. Leicht erkennbar ist die Perspektive - was auch die Fotos bestätigen, die auf die Demonstranten stürmende Polizisten zeigen - es ist die Perspektive eines am Protest Beteiligten - (1), (2), (4) und (5). Charakteristisch ist für die eher einseitige Sichtweise von konkret, dass im Unterschied zu Süddeutsche Zeitung und in geringerem Maße Neues Deutschland Aussagen von Politikem - weder in den Überschriften noch im Text - äußerst selten zitiert werden. Diese Eigenschaft kann sowohl aus der Spezifik einer Monatszeitschrift schlechthin resultieren, die meist größere und komplexere Zusammenhänge als einzelne Aussagen thematisiert, als auch von der Spezifik von konkret, die eine „Gegenzeitschrift“ mit „Gegenpositionen“ sein sollte.

Ein wesentlicher Unterschied zwischen konkret und den zwei oben analysierten Zeitungen ist ein stärkerer Akzent auf der Theorie, d.h. auf den Hintergründen und Zielen des Protests. Bei einem konkret-Leser wird ein Hintergrundwissen darüber vorausgesetzt, was eine Formulierung wie (5) ermöglicht; es ist klar, um welchen „Kampf" es sich handelt. Es braucht nicht näher definiert zu werden, wessen? wogegen? Hier reicht der bestimmte Artikel. Daraus ist zu schließen, dass die Adressatengruppe von konkret jene Zeitungsleser sind, die sich mit der APO und der Studentenbewegung identifizieren.

(1), (5) und (7) sind theoretische Texte, die die Diskussionen um das Thema „Gewalt" innerhalb der Protestbewegung widerspiegeln. (6) gehört wiederum zu jenen Texten, die die deutsche Presse selbst und ihre Beziehung zur die Studentenbewegung thematisieren. Auch hier ist die Überschrift neutral formuliert, die Anführungszeichen, in denen die Demonstrationsparole der Anti-Springer-Kampagne steht, deuten eine Distanz des Autors an. Eine genauere Analyse der Artikel würde aber eine deutliche Tendenz der „Revolutionisierung“ der Sprache (vgl. May 1983:89), die sich u.a. in der Polarisierung von politischen Begriffen, wie in den Schlüsselworten: „Gegenöffentlichkeit", „Gegengewalt", oder in den emotionalen Begriffen, z.B. „Repression“, „Establishment“, „Konsumterror“" niederschlug. Ein Einfluss der Marxschen Terminologie ist in Begriffen wie „Klassenkampf", „Herrschaft des Kapitals" leicht erkennbar. 
Vom Spiegel vom 22. April 1968 stammen folgende Überschriften zum Thema Studentenrevolte:

(1) Studenten auf den Barrikaden (Titelseite)

(2) Knüppel frei?

(3) Aufruhr. Verlorenes Wochenende

(4) Was denken die Berliner über die Studenten?

(5) Bonn. Kiesinger. Einer wird gewinnen

(6) Polizei. Gefahrenabwehr. Gesunde Vernunft

(7) „Ohne uns wäre es viel schlimmer gekommen“. SPIEGEL-Gespräch mit den SDS-Vorsitzenden Karl Dietrich Wolff und Frank Wolff

(8) Presse. Springer. Was er will

(9) Verlage. Springer. Weint euch aus

(10) Attentate. Dutschke. Der Kreidekreis

(11) Attentäter. Bachmann. Unbedingt weg

Die erste Überschrift erschien auf der Titelseite zu einem Foto von einer Menschenmenge mit Transparenten und einer roten Fahne. Obwohl es pathetisch wirkt, ist die Einstellung des Nachrichtenmagazins zu den Protesten ironisch, genauso ironisch wie seine Bewertung der staatlichen Reaktion darauf. Auf den zwei Karikaturen, die in dieser Nummer der Auseinandersetzungen zwischen den Protestierenden und der Polizei, werden beide Seiten dargestellt. Auf der einen sind ein Student und ein Polizist „die Leidtragenden“, die auf einer Tragbahre die unter Kopfschmerzen leidende „Demokratie“ tragen. Auf der anderen, bereiten sich ein Polizist und dessen Sohn auf eine Aktion - der eine schnallt sich einen Helm unter dem Kinn, der andere macht ein Transparent „Nieder mit den Etablierten!” - Unterschrift: „Generationen in Deutschland“. Der „gewaltsame Widerstand", von den radikalen Teilnehmern am Protest gepredigt, wird ,eine Schimä$\mathrm{re}^{\text {" }}$ (in: Knüppel frei?) genannt, der berittenen Polizei gilt folgender Abschnitt: „Unter dem Gewieher der Gäule keilten die grünen Dragoner mit langen Latten auf die Jugendlichen ein" (in: Gesunder Vernunft). Zwar wird die Brutalität der Polizei angeprangert, doch die ironische Wortwahl macht deutlich, dass es nicht im Ernst gemeint ist.

Den Überschriften allein ist die Ironie aber nur im Fall von (3) zu entnehmen, der Rest wirkt wegen des minimalen Informationswertes eher befremdend. Hier kommt die Werbefunktion der Überschriften deutlich zum Vorschein; sie sollen im Spiegel weder informieren wie in Süddeutsche Zeitung, noch Affekte mobilisieren wie in Neues Deutschland - ihre hauptsächliche Funktion liegt im Fesseln des Lesers. Zwar soll er wissen, wovon oder von wem der jeweilige Artikel handelt, das Unwissen aber, wie und anhand welcher Tatsachen oder Handlungen der genannten Personen, soll ihn zum Lesen des ganzen Artikels bewegen. Denn die dreifache Struktur von Überschriften (5), (6), (8), (9), (10) und (11) enthält nur folgende Elemente: was? wer? und meist ein Schlüsselwort - (10) oder 
eine Aussage - (6), (9), (11), die aber ohne Kontext erscheint und gar nicht als ein Zitat wahrgenommen wird, es sei denn von einem regelmäßigen Spiegel-Leser, dem diese Form der Überschrift vertraut ist.

Zitiert wird auch eine Aussage von einem „SDS”-Vorstandsmitglied Wolff was von einer Forum-Funktion des Magazins zeugt. Denn außer dem SDS kommt sowohl Kiesinger - (5), die westdeutsche Polizei - (6), die Gegner des SpringerVerlags - (8) wie auch der Chefredakteur von Bild - (9), selbst die Berliner in Form einer Meinungsumfrage - (4) - ein breites Spektrum von Stimmen und Perspektiven aller Parteien des Konflikts.

Sehr typisch für Der Spiegel ist die Verwendung der Umgangssprache - (2), (8), (9), (11), die auch eine Distanz zu den Ereignissen signalisiert. Als Kontrast dazu kann die pathetische Militärsprache von Neues Deutschland oder die ernsthafte Ideologiesprache von konkret dienen.

An den überdurchschnittlich großen Schlagzeilen von Bild lässt sich auch die grundlegende Tendenz der Berichterstattung dieser Zeitung erkennen. Ihre Werbefunktion hat einen besonders großen Wert für Bild, da es im Unterschied zu Süddeutsche Zeitung keine Abonnementszeitung ist und deshalb von Kioskständen aus potenzielle Käufer ansprechen muss. Außer der Größe der Buchstaben, werden daher zusätzliche graphische Mittel eingesetzt; viele Schlagzeilen werden unterstrichen oder weiß auf schwarz gedruckt. Folgende Überschriften stammen von Bild (Berlin-Ausgabe) vom 13., 16. und 17. April 1968:

(1) a) Nach dem Attentat auf Rudi Dutschke (invers)

b) Krawalle am Kudamm, Rathaus und RIAS

c) Großalarm für 20000 Polizisten

d) Terror in Berlin!

(2) Der Attentäter: „Mein Vorbild war der Mörder von Martin Luther King!"

(3) Terror nach dem Attentat - Terror nach dem Attentat (invers)

(4) Millionen bangten mit

(5) Der Attentäter: ..Lebend kriegt ihr mich nicht!"

(6) Zeitungswagen in Flammen

(7) Ku'damm wurde zum Schlachtfeld

(8) Sturm auf den Wasserwerfer

(9) Anwohner halfen mit Wassereimern

(10) Er malte Hitler und Napoleon und schoß auf Rudi Dutschke

(11) Schwere Krawalle im Bundesgebiet

(12) a) Ist das Demonstration? Ist das Diskussion? (invers)

b) Möbelhaus in Brand gesteckt (rot unterstrichen)

c) Nackte Zerstörungswut! Anschlag auf privates Eigentum

d) Marsch auf Zeitungshäuser mit Molotow-Cocktails und Stichwaffen

e) Aufrührer werden von Kommandowagen mit Sprechfunk gesteuert

(13) Fröhliche Ostern waren das!

(14) a) Ein neuer Anfang?

b) 5000 demonstrieren bei der Kundgebung unter dem Funkturm 
(15) Ärzte: Er ist recht munter

(16) a) Berlin bleibt eine Reise wert

b) Westdeutsche Besucher über die Krawalle am Kudamm

(17) 381 Festnahmen! Die Störer müssen mit Anklage rechnen

(18) Passanten halfen der Polizei

(19) Reifenstecher - es ist ein Kommunarde

(20) Und morgen das VW-Werk?

(21) Bundesgebiet: Wieder schwere Krawalle

(22) Endlich! Schnellgerichte gegen die Rädelsführer!

(23) Sagt nicht immer Studenten

(24) Statt Verlobung: Straßenschlacht!

(25) Heiße Ostern für Berlins Polizisten (invers)

(26) Mit Lärm verabschiedet

(27) ..Krähenfüße" gegen Zeitungswagen

(28) Das ist offene Rebellion gegen Demokratie (rot unterstrichen)

(29) .Er sprang vor meinen Wagen“"(rot unterstrichen)

(30) Schütz: „Macht endlich Schluss!“

Auffällig neben den genannten graphischen Besonderheiten der Bild-Überschriften sind die Ausrufezeichen, die in den aufgelisteten Überschriften $8 \mathrm{Mal}$ verwendet werden. Damit erhalten manche Schlagzeilen einen Appellcharakter (1d), oder drücken die Empörung des Autors aus - (12c), oder aber vermitteln dem Leser eine direkte Erfahrung der Ereignisse mit Rufen der Beteiligten - (5). Ein eindeutiger Appell ist auch das Zitat von Schütz - (30), (1c) trotz des fehlenden Ausrufezeichens und (23).

Sprachlich ähneln die Bild-Schlagzeilen denen von Neues Deutschland, obwohl die zwei Zeitungen politische Gegenpositionen vertreten. In beiden wird der stark emotionale Ausdruck "Terror" gebraucht, doch mit unterschiedlichen Designaten; „terroristisch“ nennt Neues Deutschland das Vorgehen der Polizei, Bild dagegen die Aktionen der Demonstranten - (1d), (3). Die Abneigung von Bild gegenüber Demonstranten drückt sich mit folgenden pejorativen Bezeichnungen aus - in bezug auf die Proteste: „Krawalle“ - (1b), (11), (21), „nackte Zerstörungswut" (12c) und in bezug auf die Protestierenden: „Rädelsführer" - (22), „Aufrührer“ (12e), ,Störer" - (17). Den Bezeichnungen von Demonstranten wird sogar ein ganzer Artikel gewidmet, nach dem das Wort „Studenten“ unpassend ist - (23).

Außer dem Wort „Terror", kommt auch der Militärwortschatz sowohl in Bild als auch in Neues Deutschland vor - "Straßenschlacht" - (24), "Schlachtfeld" - (7), "Sturm" - (8); es werden auch Waffen genannt - (12d), (27). Hier wird die These Dieckmanns von den Gemeinsamkeiten aller „Meinungssprachen“ bestätigt: Die jeweils vermittelten Inhalte wechseln mit der Ideologie; in der formalen Struktur, der Beziehung zur Wirklichkeit, der Funktion und den Funktionsweisen zeigen auch die Meinungssprachen verschiedener Systeme auffällige Ähnlichkeiten. 
Genauso wie „Terror”, wird auch das Wort „Demokratie” in den zwei Zeitungen auf unterschiedliche Parteien bezogen; ,die demokratischen Kräfte” sind nach Neues Deutschland die Protestierenden, nach Bild dagegen der Bonner Staat und der Senat von Westberlin - (28). „Demokratie" gehört zu jenen politischen Schlüsselwörtern, die jede Partei oder Organisation in bezug auf sich selbst gern benutzt und dem Gegner aus seinem Wortschatz zu entnehmen versucht. Die CDU formulierte in den Bundestagswahlen folgenden Werbespruch, auf den die SPD sehr scharf reagierte: Demokratie oder Sozialismus (vgl. May 1983:93). Dadurch wurden die zwei Begriffe in eine Entweder-Oder-Opposition gesetzt und der Begriff „Demokratie” allein für die eigene Seite beansprucht. „Demokratie”, genauso wie „Sozialismus”, „Recht”, „Freiheit” u.Ä. gehören zu den sogenannten Miranda, die Anhänger einer Organisation solidarisieren sollen. Nach Lasswell können das außer Worten auch Bilder sein, wie z.B. die von der APO gern benutzten Mao- oder Che-Guevara-Bildern (vgl. Krebs 1988:145).

Eine Sympathie für Berliner Polizisten wird dadurch deutlich, dass die Ereignisse aus ihrer Perspektive dargeboten werden - (1c), (18), (25). In (24) wird vom individuellen Schicksal eines Berliner Polizisten berichtet, der besonders „sauer" war, weil er durch die Unruhen „ein richtiges, freundliches Familienfest mit Verlobungsring und selbstgebackenem Kuchen" verpasste. Dadurch wird die Polizei nicht als eine unpersönliche Institution, sondern als eine Organisation von Menschen dargestellt, die einem typischen Bild-Leser ähnlich sind; ein Vorgang, der ein positives Image der Polizei kreiert und eine Solidarität der Leser mit den „Beamten" ermöglicht, was in (18) explizit thematisiert wird. Damit unterscheidet sich Bild wohl von Neues Deutschland, konkret und Der Spiegel, in denen auf die Brutalität der Polizei in Bild und Wort mit Nachdruck hingewiesen wird. In Neues Deutschland vom 13./14. April ist sogar unter einem Foto folgende Unterschrift zu lesen: „Polizeibüffel werden abgedrängt“. Der Gebrauch von Tiernamen als Mittel politischer Invektive wurde schon in der Nazi-Propaganda gegen Juden eingeführt. Sie wurden „Parasiten” genannt, was zugleich ein latenter Tötungsappell war (vgl. Bachem 1979:67). Neues Deutschland schreibt von einem „Polizisten mit NS-Visage”, was eine weitere aggressive Propagandatechnik ist: die der Diffamierung durch Vergleich mit einer Anti-Miranda, d.h. mit irgend einer Person oder Institution, die allgemein missbilligt oder gehasst wird. Diese Art von Diffamierung erstreckt sich auch auf Personen, die selbst zu einer AntiMiranda nicht zählen, dagegen Kontakte mit einer Un-Person und Un-Organisation unterhalten. Dadurch entsteht eine „Kontaktschuld“, die z.B. SPDPolitikern vorgeworfen wurde, die mit Kommunisten in Berührung kamen. So wurde auch Günter Grass in dem Springerblatt Berliner Morgenpost vom 14. April 1968 in einer Karikatur unter marschierenden Demonstranten als eine nebelumhüllte Gestalt gemalt, mit der Unterschrift: „Marschiert im Geiste mit". Hier wird Diffamierung durch Kontaktschuld verwendet.

Es ist typisch für Zeitungen und Zeitschriften, die die Polizei kritisieren, dass sie das mit negativen Konnotationen versehene Wort „Knüppel” gebrauchen, auch in der Verbform: „knüppeln”, „zusammenknüppeln”, substantivisch in ei- 
nem Kompositum „Knüppelgarde“, Neues Deutschland oder selbst adjektivisch: „zusammenknüppelbare Demonstrationen”, konkret. Regierungsnahe Presse dagegen berichtet von „Einsatz von Schlagstöcken”, oft mit der Kategorie der Notwendigkeit (vgl. Jansen 1968:132): „Die Polizei musste zum Schlagstock greifen", Bild. Dadurch wird suggeriert, dass die Polizei in Notwehr zum Schlagstock, bzw. zur Knüppel griff und wird z.T. von der Verantwortung für die Folgen entlastet.

Den Schlagzeilen (6), (19), (27) und am deutlichsten (12c) lässt sich entnehmen, dass nicht der Protest selbst im Vordergrund steht, sondern die Sachschäden, die dabei entstanden. Es werden ausführlich äußere Erscheinungen des Protests dargestellt, seine Inhalte werden aber ähnlich wie in Süddeutsche Zeitung und Neues Deutschland verschwiegen. Aus der Lektüre von Bild könnte man schließen, die Ausschreitungen waren „nackte Zerstörungswut”, ohne jeglichen politischen Hintergrund. Was in konkret politisiert wird, indem man im Zusammenhang mit den Osterereignissen in Deutschland Parallelen zur "Gewaltherrschaft im Kapitalismus” und „Ausplünderung der Kolonialländer” zieht (in: Gewalt), wird in Bild entpolitisiert.

Die Mehrheit der Bild-Schlagzeilen enthält eine sehr kritische Wertung der Demonstrationen, was in Anbetracht der Anti-Springer-Kampagne mit ihren Losungen wie „Killt Bild" nicht verwundern darf. Es gibt aber auch neutrale Formulierungen, die auch in einer Studentenzeitschrift denkbar wären - (8) oder (14). Eine Schlagzeile drückt sogar Sympathie für den schwerverletzten Rudi Dutschke - (4). Weiter ist im Artikel zu lesen: ,politische Gegnerschaft wird überdeckt durch Anteilnahme am menschlichen Schicksal".

Wie Der Spiegel greift Bild häufig zu umgangssprachlichen Wendungen, insbesondere in den Zitaten - (5), (15), (29), (30). Von der Sprache der Studentenbewegung stammt nur ein Wort aus der Überschrift (19) - „Kommunarde” allerdings in einem Zusammenhang, der für die Kommunarden eine sehr schlechte Werbung ist. Aus der syntaktischen Struktur der Überschrift kann gefolgert werden, dass die Zugehörigkeit zu einer Kommune Grund für das Reifenaufschlitzen war.

Von einer ,systematischen Hetze" aber, in der die Studenten die Ursache des Mordversuchs an Dutschke sahen, ist es aber weit entfernt. Nur ein Foto, eine Nahaufnahme von Fritz Teufel unter anderen Demonstranten, könnte eventuell unter diese Kategorie fallen. Darunter lautet die Unterschrift: „....und hin und wieder in der Menge ein bekanntes Gesicht, wie hier - Fritz Teufel". Unklar ist, mit welcher Absicht auf den Kommunarden hingewiesen wird. Ein ähnliches Foto von Dutschke wurde in Bild vom 28. November 1967 veröffentlicht, auf dem er mit einem Stern markiert war. Dass diese Fotos die Funktion von Fahndungsfotos hatten, lässt sich aber nicht beweisen.

Rudi Dutschke starb an den Spätfolgen des Attentats 1979. 2001 griff der langjährige Freund von Dutschke, Wolf Biermann, das Thema auf: „Immerhin 
war es vor allem die Hetze in Springers Bild-Zeitung in den heißesten Zeiten des Kalten Krieges, die in West-Berlin eine Stimmung in der Bevölkerung aufheizte, ohne die der junge Nazi Bachmann wohl kaum seine drei Kugeln in den Kopf von Rudi Dutschke geschossen hätte." (zit. nach Koelbl) Der Liedermacher der Protestbewegung schieb diesen Text für die Springerzeitung Die Welt.

\section{LITERATUR}

Bachem, Rolf (1979): Einführung in die Analyse politischer Texte. München.

Barańczak, Stanisław (1983): Czytelnik ubezwlasnowolniony. Paris.

Bergsdorf, Wolfgang (1978): Politik und Sprache. München.

Borgosz, Józef (1971): Bunt mlodzieży na Zachodzie. Geneza - charakter - ideały. Warszawa

Bralczyk, Jerzy (1987): O języku polskiej propagandy politycznej lat siedemdziesiatych. Upsala.

Brandt, Karl-Werner (1986): Aufbruch in eine andere Gesellschaft: neue soziale Bewegungen in der Bundesrepublik. Frankfurt/Main.

Bröder, Friedrich (1969): Ein Sprachrohr des Rechtsradikalismus. Mainz.

Broński, Maciej (1981): Totalitarny język komunizmu. In: Teksty i preteksty. Paris.

Bude, Heinz; Kohli, Martin (1989): Radikalisierte Aufklärung. Studentenbewegung und Soziologie in Berlin 1965 bis 1970. München.

Daniel, Ute; Siemann, Wolfram (Hrsg.) (1994): Propaganda. Meinungskampf, Verführung und politische Sinnstiftung 1789-1989. Frankfurt/Main.

Diesinger, Gerald; Gries, Rainer (Hg.) (1996): Propaganda in Deutschland. Zur Geschichte der politischen Massenbeeinflussung im 20. Jahrhundert. Darmstadt.

Dobek-Ostrowska, Bogusława; Fras, Janina; Ociepka, Beata (1997): Teoria i praktyka propagandy. Wroclaw.

Ellwein, Thomas (1989): Krisen und Reformen. Die Bundesrepublik seit den sechziger Jahren. München.

Garlicki, Bolesław (1981): Selekcja informacji w dziennikarstwie. Kraków.

Glotz, Peter; Langerbucher, Wolfgang (1969): Der missachtete Leser. Köln.

Good, Colin (1985): Presse und soziale Wirklichkeit. Düsseldorf.

Grosser, Alfred (1970): Geschichte Deutschlands seit 1945. München.

Gudorf, Odilo (1981): Sprache als Politik. Köln.

Halter, Hans (1999): „Bürger, laßt das Gaffen sein!“. Die Apo und die Große Koalition. In: Der Spiegel 20/1999-21. Mai 1999.

Happenkamps, Hermann (1977): Information oder Manipulation? Tübingen.

Hundhausen, Carl (1975): Propaganda. Essen.

Jansa, Axel (1999): Pädagogik, Politik, Asthetik: Paradigmenwechsel um „68. Frankfurt/Main.

Jansen, Berndt; Klönne Arno (Hg.) (1968): Imperium Springer. Macht und Manipulation. Köln.

Kaltenbrunner, Gerd-Klaus (Hg.) (1976): Die Macht der Meinungsmacher. Die Freiheit zu informieren und informiert zu werden. München.

Kamińska-Szmaj, Irena (1994): Judzi, zohydza, ze czci odziera - język propagandy politycznej $w$ prasie 1919-1923. Wrocław.

Karpiński, Jakub (1989): Mowa do ludu. Szkice o jezzyku polityki. Warszawa.

Klemperer, Viktor (1947): LTI. Notizbuch eines Philologen. Berlin.

Koelbl, Susanne (2001): Wer war mein Vater?.In: Der Spiegel 7/2001 - 12. Februar 2001.

Kossecki, Józef (1972): Socjotechnika propagandy politycznej. In: Zeszyty Prasoznowcze, Nr. 3/1972.

Kot, Wiesław (1999): Lata 70. Od propagandy sukcesu do narodzin Solidarności. Poznań 1999.

Krause, Christian; Lehnert, Detlef; Scherer, Klaus-Jürgen (1980): Zwischen Revolution und Resignation. Bonn. 
Kraushaar, Wolfgang (1999): 1968, das Jahr der Rebellion. In: Der Spiegel 13/1999.

Krebs, Mario (1988): Ulrike Meinhof. Ein Leben im Widerspruch. Hamburg.

Kwiatkowski, Stanisław (1977): Stowo i emocje w propagandzie. Warszawa.

Langguth, Dieter (1972): Z badań nad oddziaływaniem prasy w NRD. In: Zeszyty Prasoznowcze Nr. $1 / 1972$.

Linden, Peter (2000): Wie Texte wirken: Anleitung zur Analyse journalistischer Sprache. Berlin 2000.

Lippmann, Walter (1960): Public Opinion. New York.

Mackensen, Lutz (1968): Einige Bemerkungen über das Kraftfeld zwischen Sprache und Massenmedien. In: Deutsche Studien, März 68.

Mager, Friedrich; Spinnarke Ulrich (1968): Was wollen die Studenten? Frankfurt/Main.

May, Iwona (1983): Język a polityka - Język niemiecki w RFN. In: Przeglqd Zachodni 1983/3.

Meyer, Gerd (1968): Warum protestiert die Jugend? In: X-informationen. 1968/40.

Mrozowski, Maciej (1991): Między manipulacja a poznaniem. Warszawa 1991.

Noelle-Neumann, Elisabeth (Hg.) (1989): Fischer Lexikon. Publizistik, Massenkommunikation. Frankfurt/M.

Ortlieb, Heiz-Dietrich (1968): Die missverstandene Revolte. Hamburg.

Pisarek, Walery (1976): Język sluży propagandzie. Kraków.

Reich, Hans (1968): Sprache und Politik. Untersuchungen zu Wortschatz und Wortwahl des offiziellen Sprachgebrauchs in der DDR. München.

Schopenhauer, Arthur (1976): Erystyka czyli sztuka prowadzenia sporów. Kraków.

Sztumski, Janusz (1980): Propaganda polityczna. Jej problemy i metody. Katowice.

Sztumski, Janusz (1990): Propaganda - jej problemy i metody. Katowice.

Szymoński, Rafał (1988): O urbanowszczyżnie i inmych metodach manipulacji. Katowice.

Thomason, Oliver (2001): Historia propagandy. Warszawa.

Tomczak, Maria (1984): Przemoc i polityka. Poznań.

Varga, Karoly (1972): Wiadomości zagraniczne. In: Zeszyty Prasoznowcze Nr. 3/72.

Wagińska-Marzec, Maria (1996): Protest-song w Republice Federalnej Niemiec w okresie kontestacji mlodzieżowej. Poznań.

Wandowski, Henryk (1971): Axel Springer - „król “ prasy zachodnioniemieckiej. Warszawa.

Wenger, Klaus (1988): Kommunikation und Medien. München.

Wojtasik, Lesław (1979): Agitacja polityczna. Poradnik dla agitatora. Warszawa.

Wojtasik, Lesław (1986): Psychologia propagandy politycznej. Warszawa.

Zimmer, Dieter (1977): Wörter und Waffen. Politik als Sprachkampf. In: Die Zeit 21.10.77. 